\title{
THERMAL-HYDRAYULICS AND NEUTRONICS CODES COUPLING FOR THE ANALYSIS OF A LEAD FAST REACTOR
}

\author{
G. Barbi*, A. Chierici*, V. Giovacchini ${ }^{* \dagger}$, M. Lanconelli* and S. Manservisi* \\ *Alma Mater Studiorum - University of Bologna, Department of Industrial Engineering (DIN) \\ Via dei Colli 16, Laboratorio di Montecuccolino, 40136, Bologna, Italy \\ †email: valentin.giovacchin2@unibo.it
}

Key words: Thermal-Hydraulics, Neutronics, Lead-cooled Fast Reactors, Multiphysics

\begin{abstract}
In this work the thermal-hydraulics and neutronics behavior of a Lead Fast Reactor (LFR) core is investigated evaluating the power generation distribution taking into account the local temperature field. The temperature field is evaluated using the CFD finite element code FEMuS and exchanged with the multiscale neutron code DONJON-DRAGON, which interpolates the macroscopic cross-sections according to the local temperature field and local lead density distribution. As a result, the neutron flux changes and defines a new power density distribution which is used to update the temperature field into the CFD code. The coupling between neutron and CFD codes is achieved through their inclusion into the numerical platform SALOME. The numerical libraries MED, included into the SALOME platform, are used to exchange data run-time between FEMuS and DONJON.
\end{abstract}

\section{INTRODUCTION}

The modeling of nuclear reactor systems is one of the most challenging tasks due to the many different physics involved, i.e. neutron transport, fluid dynamics, and heat transfer. In the active region of the core, neutrons interact with nuclear materials and the coolant. Neutron cross-sections in these materials depend on the local temperature and density, while the heat released by fission events depends on the neutron fission cross-section.

The mutual interaction between the neutron flux and the local temperature field is a crucial aspect in the study of a reactor core. In this work, the solution of a neutron code is coupled with the solution of a CFD code to investigate the thermal-hydraulics dynamics of a Lead-cooled Fast Reactor (LFR) taking into account the dependence of the power generation on the temperature distribution. With this approach, it is possible to take advantage of already validated codes and expertise developed in years. The CFD module used in this work is the in-house multi-grid finite element code FEMuS developed at the 
University of Bologna [1], which has been validated for the thermal-hydraulics simulation of a reactor core in $[2,3]$. FEMuS code contains solvers for incompressible Navier-Stokes equations, heat transfer, turbulence models, Fluid- Structure Interaction problems, multiphase flows and optimal control with the adjoint method. The neutron code used has been developed at the École Polytechnique de Montréal and it is composed of the two modules DRAGON [4] and DONJON [5]. The computer code DRAGON is a lattice code designed around solution techniques of the neutron transport equation. The two main components of the code DRAGON are its multigroup flux solver and its one-group collision probability $(\mathrm{CP})$ tracking modules. DONJON is a full-core modelization code designed around solution techniques of the neutron diffusion or simplified $P_{n}$ equation. The DRAGON modules are used with DONJON code to define the reactor geometry, provide the macrosconic cross-section libraries and perform micro-depletion calculations.

The coupling between the neutron and CFD code is achieved through their inclusion into the numerical platform FemusPlatform based on the SALOME project. The numerical platform [6] has been developed as an environment where several numerical codes can be run together, allowing to model complex physical phenomena on different physical scales. All the included codes can exchange data runtime thanks to the MEDMem libraries, a computational toolbox integrated into SALOME that handles the exchange

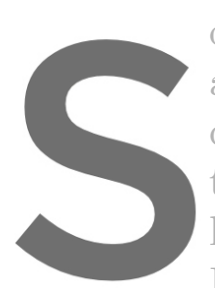
of data between codes a PWR nuclear readt codes [7], showing the to investigate the thert hexagonal geometry European Demonstrat
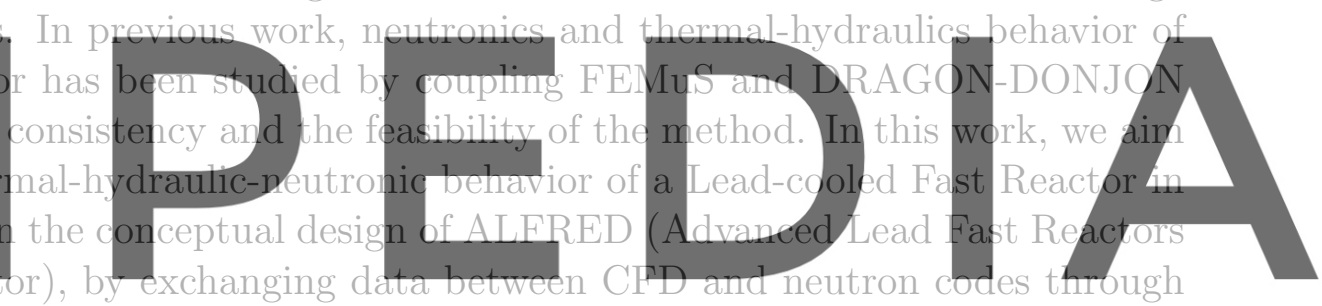

the MEDMem interfaces

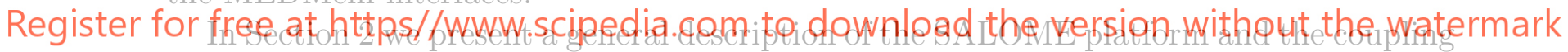

procedure. In Section 3 the multiphysics modeling of the LFR core is described and some numerical results are presented and discussed. Finaliy, in the last section, we draw some conclusions and future perspectives.

\section{Numerical Platform}

SALOME is an open-source platform for preprocessing and postprocessing of numerical simulations, developed by CEA and EDF. SALOME integrates tools for preprocessing CAD applications and for generating meshes. With the GEOM module one can create CAD models that can be meshed using the MESH module, which provides the possibility to create, modify, import and export meshes in various formats. SALOME offers also the means to solve complex multiscale and multiphysics problems by exchanging information among different codes, through the MED module. The MED module provides a library for storing and recovering data in MED format, associating numerical meshes and fields allowing the exchange between different codes and solvers. Three main libraries are provided: MED File, MED Memory, and MED CORBA. The MED File is a $\mathrm{C}$ and 
Fortran API that implements functions for reading and writing fields and meshes into files with .med extension. The MED memory is a $\mathrm{C}++$ and Python API that allows storing mesh and field objects in memory. A numerical field can then be read from a file, using the MED file library, and stored in memory by using the MED memory library. Finally, the MED CORBA is an API written to facilitate the distributed computation inside SALOME platform, providing functions for data accessing.

The integration of a code into the SALOME platform is obtained by generating an interface with functions available in the MEDMem library that allow the data transfer from the platform to the code and vice-versa. The integration of an open-source code into the numerical platform can be divided into three major steps: the first is the generation of the code-library from the original code, the second is the generation of the MEDMem interface and finally the generation of SALOME-code interface integration.

The first step for the thermal-hydraulics and neutronic analysis of the Lead-cooled Fast Reactor is the creation of data structures containing the macroscopic cross-sections of the elements forming the nuclear reactor fuel. The creation of a macroscopic crosssections database is performed by using the lattice code DRAGON. The comprehensive neutronic behavior is investigated with DONJON, a full core simulation code, which

uses the nuclear properties evaluated using the lattice code DRAGON to compute the

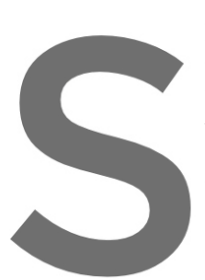
macroscopic flux di

hydraulies analysis the SAIOME platf code, the new peak FEMrs and the new on the neutronic flux dis
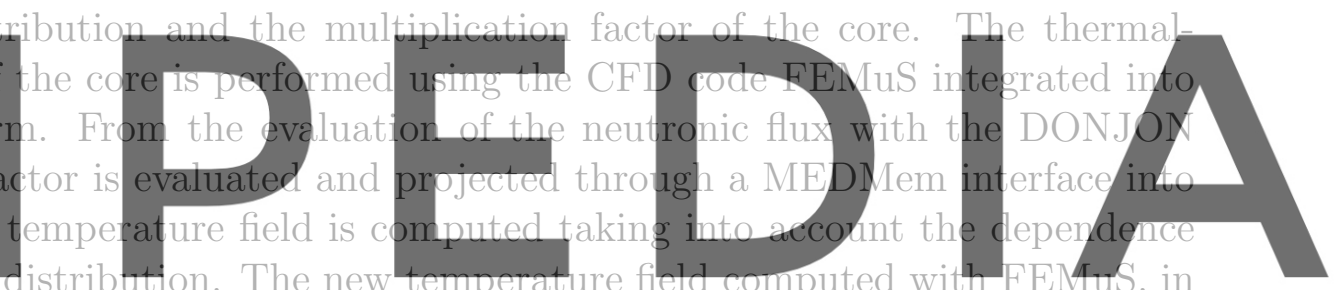

its turn, is exchanged with DONJON through the MEDMem interface and the material

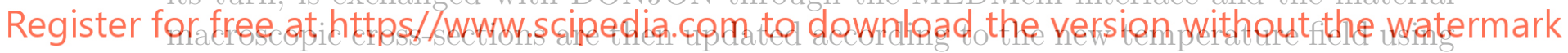
the database generated with DRAGON.

\section{$3 \quad$ Numerical Simulations}

In this section, we show numerical results obtained by coupling the neutronic code DONJON with the thermal-hydraulics code FEMuS. The data exchange is gained through MED interfaces. During a time step iteration, the thermal-hydraulics code evaluates the temperature field according to a given distribution of energy production. The temperature field is then exchanged with the neutronic code which updates the macroscopic crosssections of materials using COMPO object generated with DRAGON and the consequent neutron flux is computed. The new peak factor is evaluated and projected with the MEDMem interface into FEMuS code that evaluates the new temperature field. 

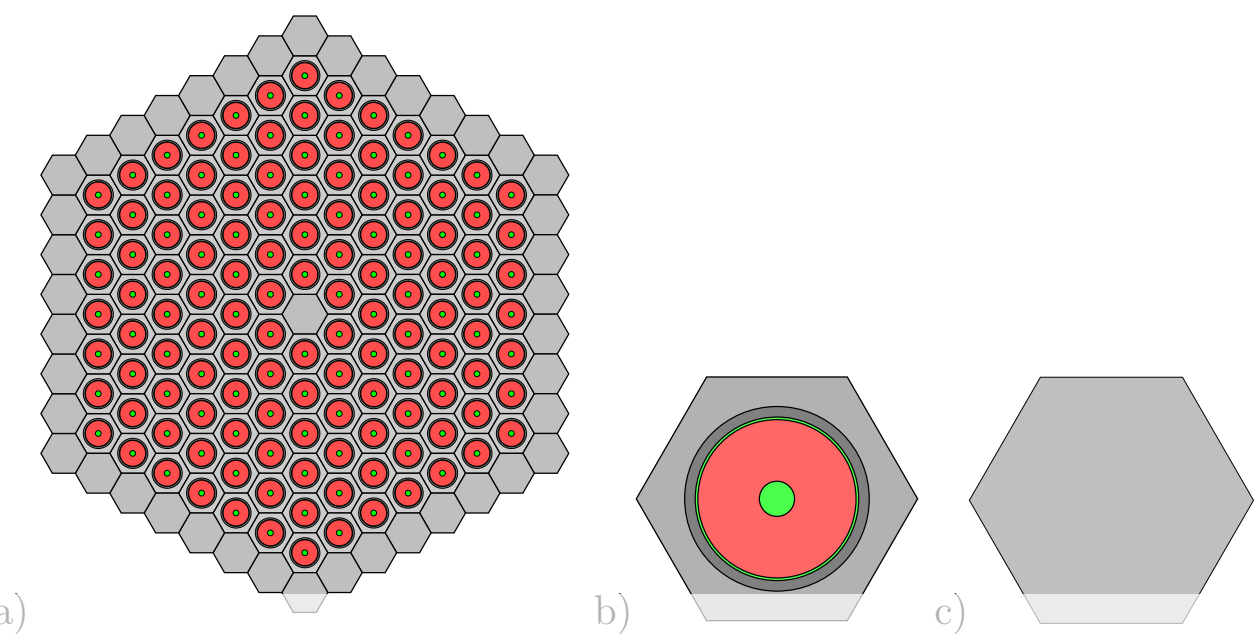

Figure 1: a) A fuel assembly with 126 fuel pins. b) The cell represents fuel pins constituted by lead, cladding, helium gap and nuclear fuel, c) the cell represents the structural part of the fuel assembly.

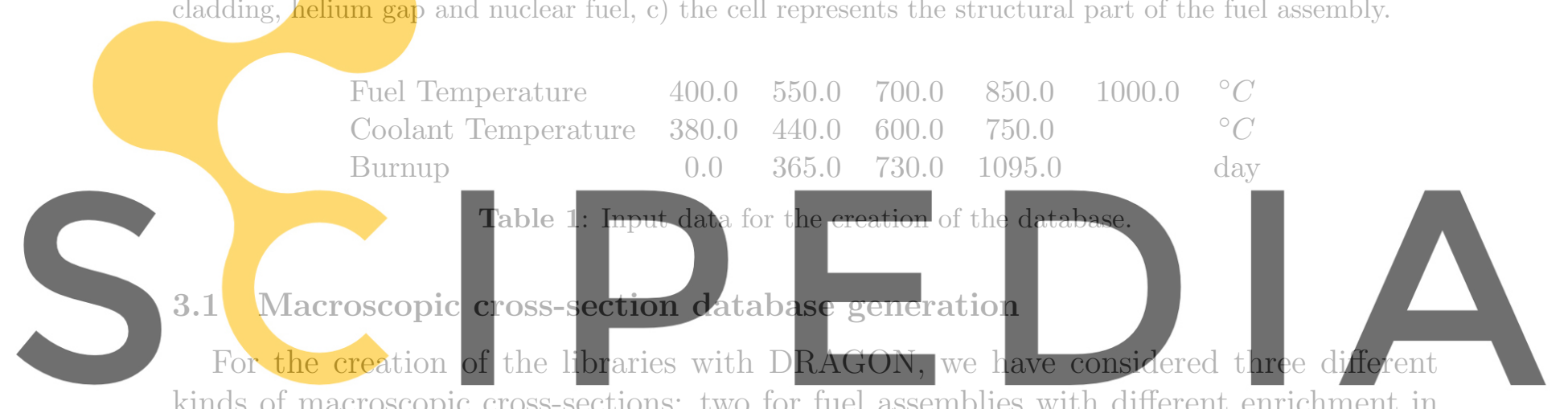

kinds of macroscopic cross-sections: two for fuel assemblies with different enrichment in

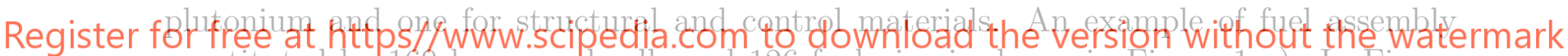
constituted by 169 hexagonal cells and 126 fuel pins is shown in Figure 1 a). In Figure

1 b) a fuel pin composed of lead, cladding, helium gap, and nuclear fuel with different enrichment in plutonium is represented, while in Figure $1 \mathrm{c}$ ) a hexagonal cell of the fuel assembly containing only structural elements is shown. To compute the macroscopic crosssections, we use JEFF 3.1.2 microscopic cross-section library for the nuclear data with 315 energy groups. The neutron transport equation in the lattice code is solved with the collision probability method and the data structures of the macroscopic cross-sections are created taking into account self-shielding, fuel burn-up, temperature and concentration changes. The macroscopic cross sections are evaluated for different fuel compositions (enrichment inner MOX 20.5\% and outer MOX 26.2\%), different fuel, different coolant temperatures and different burnup, as reported in Table 1 . The generation of macroscopic cross-section library depends only on the fuel pin composition and geometry and the possibility to evaluate the cross-section in a range of compositions and temperatures is meant for avoiding the generation of this library every time iteration during a simulation. DRAGON generates the macroscopic properties and stores them in COMPO files where 
the data structures are condensed in 33 energy groups to reduce the computation time.

\subsection{Neutronic and Thermal-Hydraulics coupling}
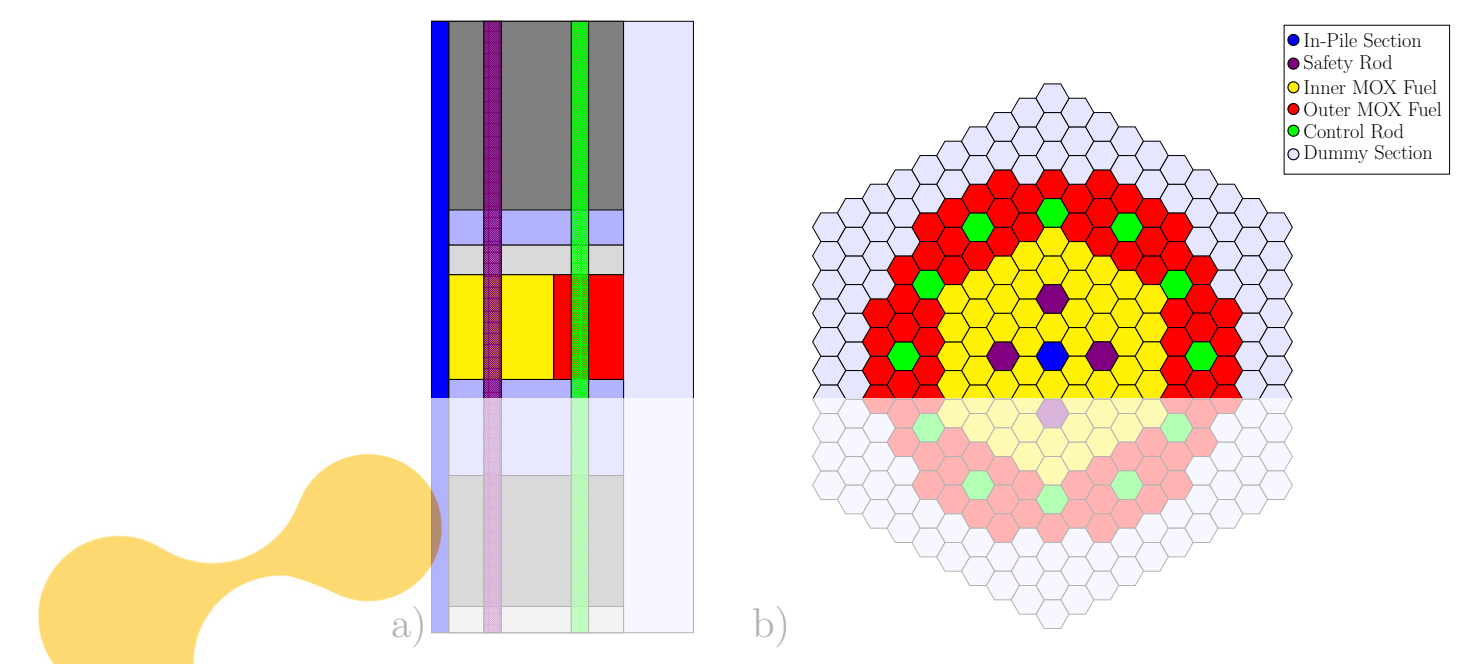

Figure 2: a) Axial profile and b) core map of the reactor composed by assemblies of In Pile section (blue), Safety rods (purple), control rods (green), Inner MOX Fuel (yellow), Outer MOX Fuel (red) and

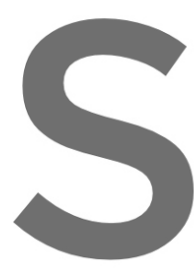

Dummy elements (gray
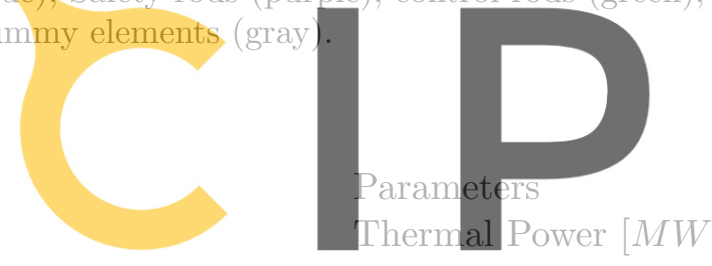

Total Fuel assembly
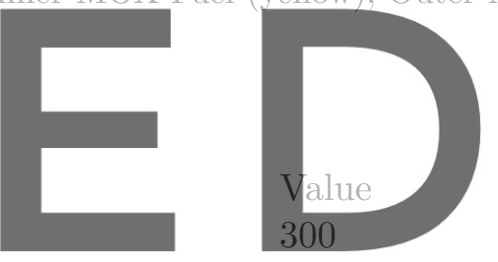

134

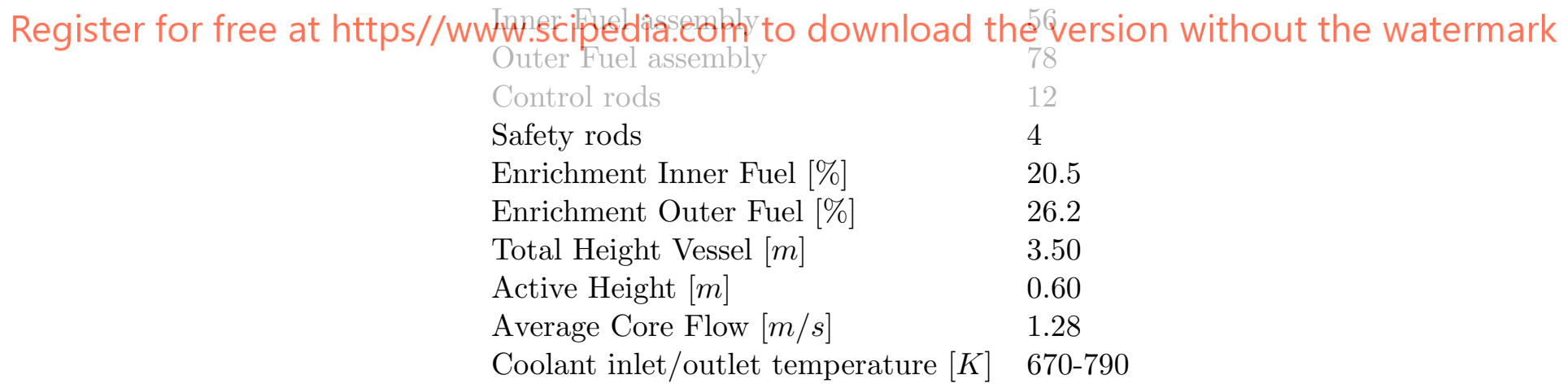

Table 2: Reference data used to model ALFRED reactor.

The comprehensive neutronic behavior is investigated by using the DONJON code which solves the multigroup diffusion problem. Since DONJON can handle only cartesian structured mesh, the geometry of the whole reactor has been considered as a hexagonal prism as reported in the axial profile (a) and the core map (b) of Figure 2. To consider the real shape of the active region of the core, dummy elements have been introduced 
a)

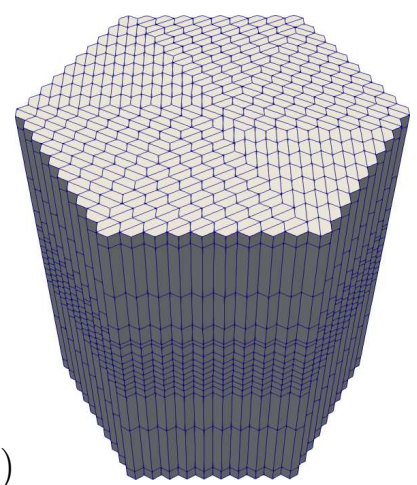

b)

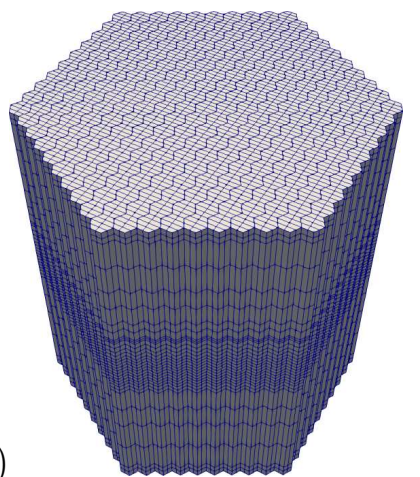

c)

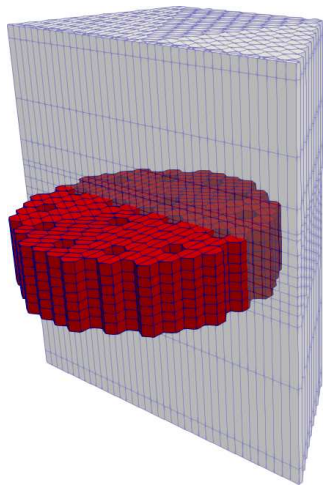

Figure 3: a) DONJON and b) FEMuS hexagonal meshes, in c) the active zone of the core is in red.

representing the material where the neutron flux is zero and is not evaluated, as reported in gray in Figure 2a) and b). The geometry data and parameters of the core are reported in Table 2. The MOX fuel assemblies present different enrichment for the inner $(20.5 \%)$ and outer zone (26.2\%), reported respectively in yellow and in red in Figure 2 a) and b). Safety rod and control rod assemblies are shown in Figure 2 a) and b) in purple and green respectively. The reactor is characterized by a total height of $3.50 \mathrm{~m}$, while the active part

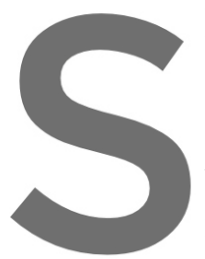
height is $0.60 \mathrm{~m}$.

The thermal-hydra FEMuS. In the core

the velocity, pressure is necessary. The of which is made un of 126 fuel and the
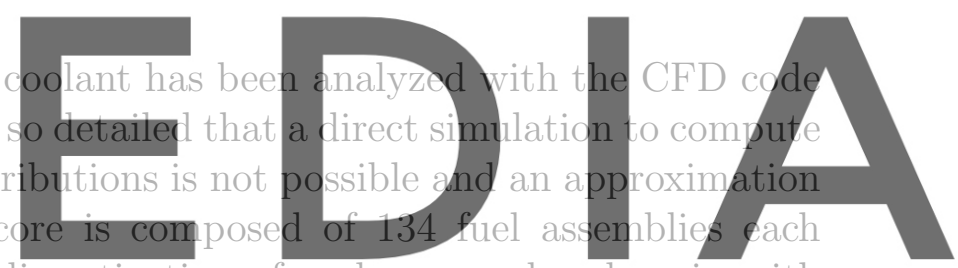

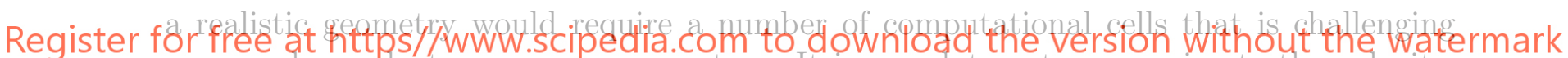
even on huge clusters or supercomputers. It is mandatory to approximate the velocity, pressure and temperature fields inside the core on a coarser grid of computational cells that homogenize the underlying structures of pin and sub-channels. At the fine level, the fluid motion is exactly resolved by the pressure, velocity and temperature solution field, while the coarse level takes into account only large geometrical structures and solves only for average fields. Instead of simulating each flow sub-channel between the pins, the core is considered to be a porous media, where average velocity and temperature fields are computed. For more details on this approach, the reader can consult [8]. Let $r$ be the porosity coefficient defined as $r=A_{c} / A_{f a}$ where $A_{c}$ is the coolant flow area and $A_{f a}$ be the total section of the fuel assembly. The conservation equations can be written as

$$
\begin{aligned}
& \nabla \cdot(r \rho \hat{\mathbf{u}})=0, \\
& \frac{\partial(r \rho \hat{\mathbf{u}})}{\partial t}+\nabla \cdot(\rho r \hat{\mathbf{u}} \hat{\mathbf{u}})=-\nabla \hat{p}+\nabla \cdot r \hat{\boldsymbol{\tau}}^{e f f}+\hat{m}_{g}, \\
& \frac{\partial(r \rho c \hat{T})}{\partial t}+\nabla \cdot(r \rho c \hat{\mathbf{u}} \hat{T})=\nabla \cdot\left(r \lambda^{e f f} \nabla \hat{T}\right)+r Q_{f}+\hat{q}_{g},
\end{aligned}
$$


where $\hat{m}_{g}$ and $\hat{q}_{g}$ are additional source terms deriving from the porous medium hypothesis. The subgrid momentum source term $\hat{m}_{g}$ represents the loss of pressure in the coolant due to the viscous friction in sub-channels, while the subgrid heat source $\hat{q}_{g}$ represents the heat generated through the fuel pin surface.

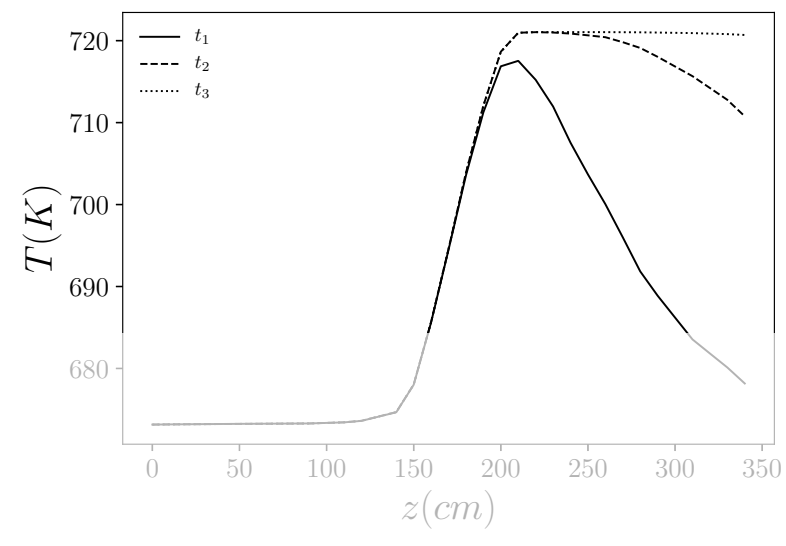

Figure 4: Temperature profile over the center line of the domain at different time steps.

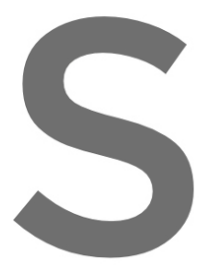

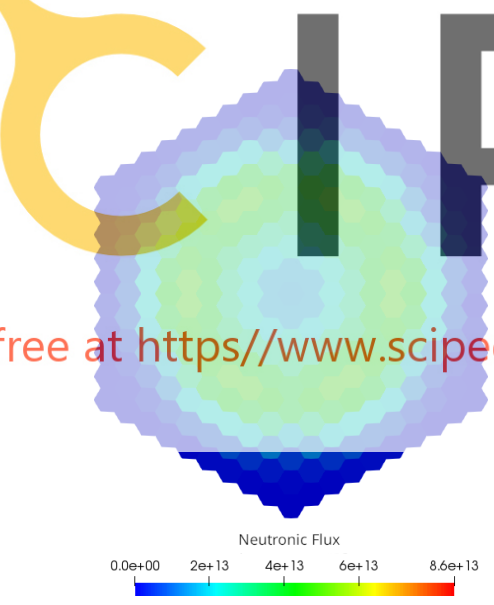

a) $z=150 \mathrm{~cm}$
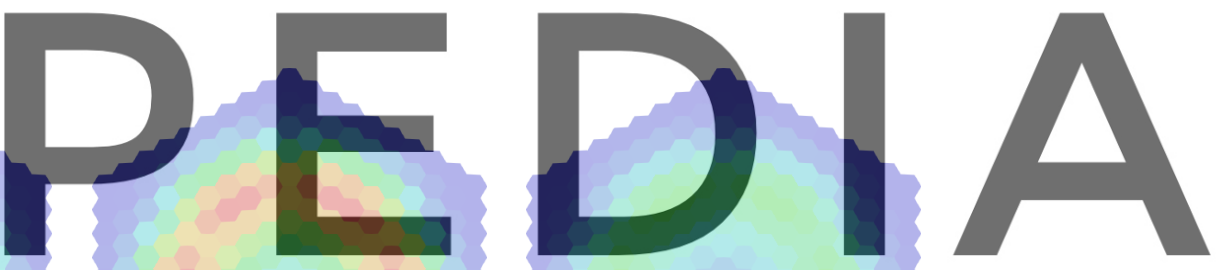

Register for free at https//www.scipedia.com to download the version without the watermark

Figure 5: Neutron flux distribution over a section of the core at a) $150 \mathrm{~cm} \mathrm{b)} 175 \mathrm{~cm}$ c) $200 \mathrm{~cm}$ from the bottom.

The interface between the neutron and CFD modules is created by the construction of a duplicated mesh of the two computational domains in MEDMem format and creating a map between them. In Figure 3 a) the mesh used for DONJON and in Figure 3 b) the one used for FEMuS are shown. The two meshes have the same geometry but different refinements. In Figure $3 \mathrm{c}$ ) the active zone of the reactor code is shown in red. 


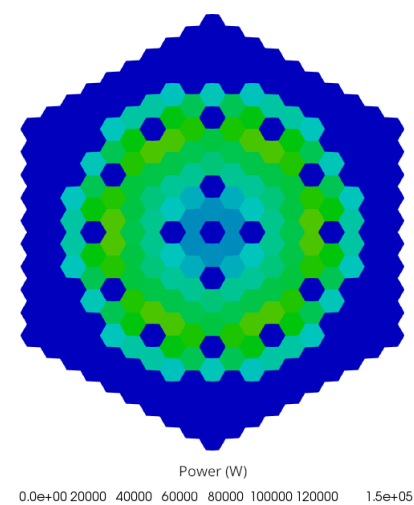

a) $z=150 \mathrm{~cm}$

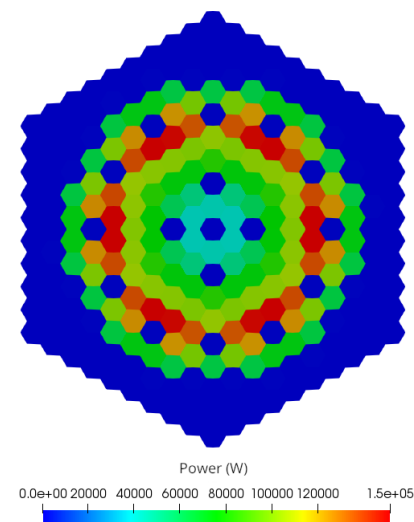

b) $z=175 \mathrm{~cm}$

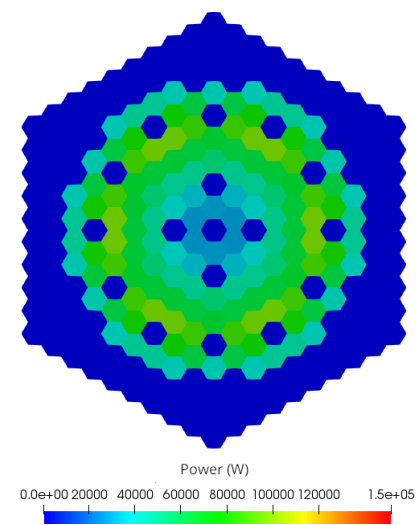

c) $z=200 \mathrm{~cm}$

Figure 6: Power distribution over a section of the core at a) $150 \mathrm{~cm}$ b) $175 \mathrm{~cm}$ c) $200 \mathrm{~cm}$ from the bottom.
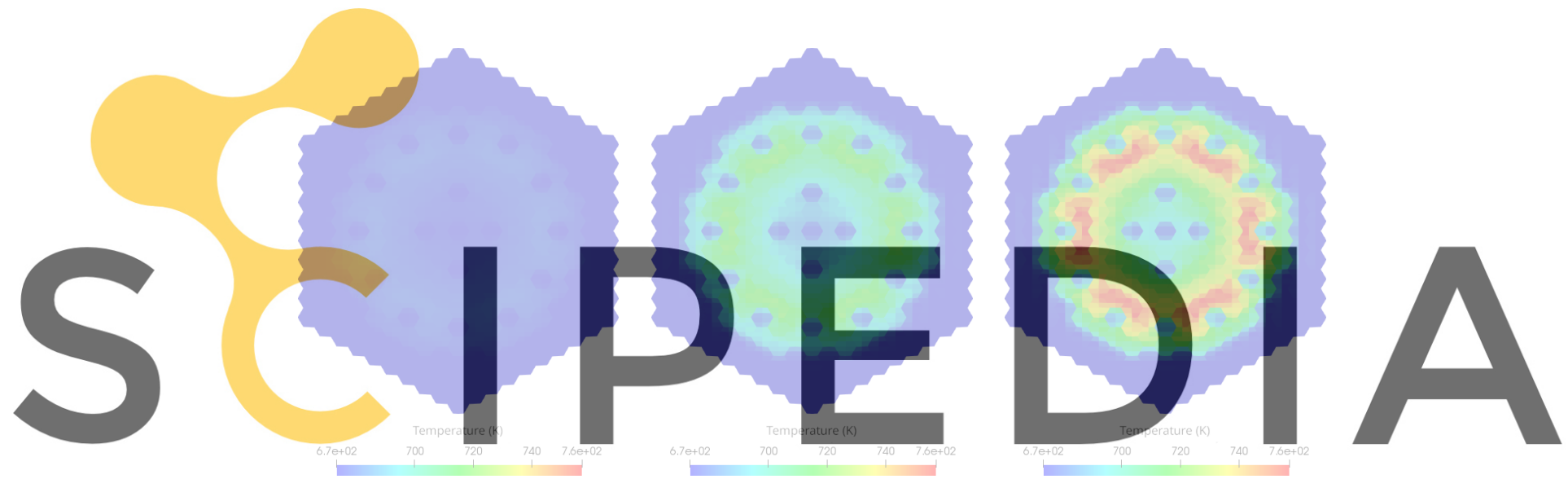

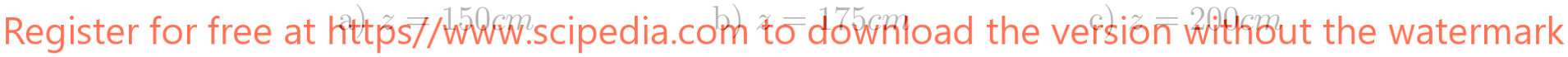

Figure 7: Temperature field over a section of the core at a) $150 \mathrm{~cm}$ b) $175 \mathrm{~cm} \mathrm{c)} 200 \mathrm{~cm}$ from the bottom.

\subsection{Numerical Results}

The temperature field initial value is set equal to $670 \mathrm{~K}$ and the axial velocity field to a constant value of $1.28 \mathrm{~m} / \mathrm{s}$. In Figure 4 the temperature field over the center-line of the domain at different iteration steps $\left(t_{1}, t_{2}, t_{3}\right)$ is shown. The temperature field increases in the active zone of the core $(150-210 \mathrm{~cm})$ due to the energy production in the initial steps $t_{1}$ and $t_{2}$ and then all the upper zone is heated until the steady-state is reached $\left(t_{3}\right)$. The steady solution is obtained after several iterations between the thermal-hydraulic and neutronic code with temperature and density corrections.

Figure 5 shows the total neutron flux over three different sections of the reactor defined by $z$-value of $150 \mathrm{~cm}, 175 \mathrm{~cm}$ and $200 \mathrm{~cm}$. The neutron flux is computed with DONJON using 33 energy groups and the integral over all the energy values is reported. The power distribution over the sections of the core reactor at $150 \mathrm{~cm}, 175 \mathrm{~cm}$ and $200 \mathrm{~cm}$ is reported in Figure 6. The thermal power is computed based on peak factors representing the ratio 
between the assembly thermal source and the average assembly power. Figure 7 shows the temperature field over the sections of the reactor defined by $z$-value of $150 \mathrm{~cm}, 175 \mathrm{~cm}$ and $200 \mathrm{~cm}$. The temperature distribution shows the cooled area in the assemblies where there is no heat generation.

\section{CONCLUSIONS}

In this work, the thermal-hydraulics-neutronics analysis of a Lead-cooled Fast Reactor on the conceptual design of ALFRED has been presented. The multiphysics behavior of the nuclear reactor core has been investigated integrating the thermal-hydraulics code FEMuS and the neutron code DRAGON-DONJON into the numerical platform FemusPlatform, based on the SALOME project. Thanks to MEDMem libraries, a MEDMem interface has been created for the data exchange between the two codes. At every time iteration, a new power distribution is evaluated with DONJON and projected into FEMuS for the solution of the new temperature field taking into account the dependence on the neutron flux distribution. The new temperature field computed with FEMuS is then exchanged with DONJON, the material macroscopic cross-sections are updated and a new neutron flux distribution is evaluated. The coupling algorithm between the two codes stops when a steady solution is reached. The numerical results presented have shown the consistency and robus

it is possible to increasc platform. It would be

for the simulation of th and neutronics analys

nuclear power plant with a multiphysics an
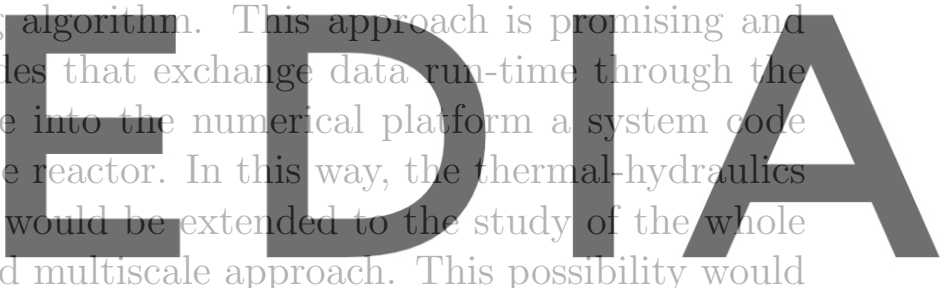

Register for free at https//wWw.scipedia.com to download the version without the watermark REFERENCES

[1] https://github.com/FemusPlatform/femus - Official repository for multigrid Finite Element Code FEMuS.

[2] Bornia, Giorgio and Finelli, Marco and Manservisi, Sandro and Mikhin, Valeri and Polidori, Massimiliano and Voukelatou, Nadia. Development and validation of femlcore code for the thermal hydraulics of open cores.

[3] Bornia, Giorgio and Cerroni, Daniele and Manservisi, Sandro and Polidori, Massimiliano and Donato, Filippo. FEM-LCORE Code: parallelization, turbulence models and code integration Technical Report ENEA-UNIBO 2012. 9, 10, 11, 17. (2012)

[4] Marleau, Guy and Hébert, Alain and Roy, Robert. A user guide for DRAGON Version 4. Institute of Genius Nuclear, Department of Genius Mechanical, School Polytechnic of Montreal (2011). 
[5] Hébert, A and Sekki, D and Chambon, R. A user guide for DONJON version 4. École Polytechnique de Montréal Montréal QC, Canada, Tech. Rep. IGE-300 (2013).

[6] https://github.com/FemusPlatform/NumericPlatform - Official repository of opensource code based Numerical Platform.

[7] Cerroni, Daniele and Da Vià, Roberto and Manservisi, Sandro and Menghini, Filippo and Scardovelli, Ruben. CFD and Neutron codes coupling on a computational platform. Journal of Physics: Conference Series. (2017).

[8] Cervone, Antonio and Manservisi, Sandro. A Three-Dimensional CFD Program for the Simulation of the Thermo-Hydraulic Behaviour of an Open Core Liquid Metal Reactor.
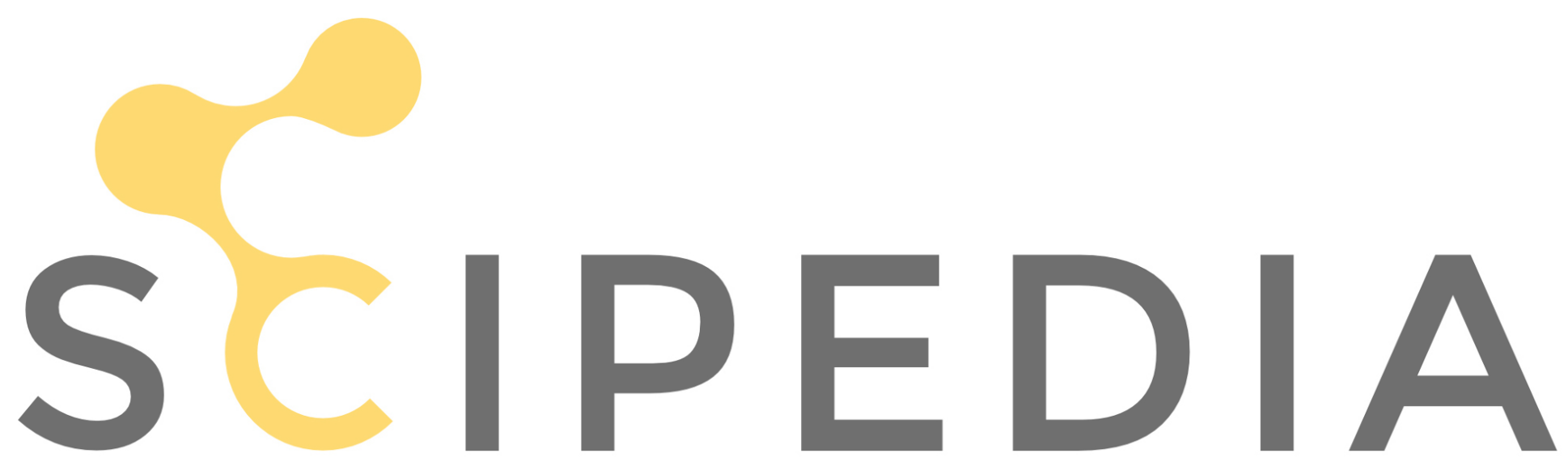

Register for free at https//www.scipedia.com to download the version without the watermark 